\title{
MODIFIED STREAMLINE DIFFUSION SCHEMES FOR CONVECTION-DIFFUSION PROBLEMS
}

\author{
YIN-TZER SHIH* AND HOWARD C. ELMAN ${ }^{\dagger}$
}

\begin{abstract}
We consider the design of robust and accurate finite element approximation methods for solving convection-diffusion problems. We develop some two-parameter streamline diffusion schemes with piecewise bilinear (or linear) trial functions and show that these schemes satisfy the necessary conditions for $L^{2}$-uniform convergence of order greater than $1 / 2$ introduced by Stynes and Tobiska. For smooth problems, the schemes satisfy error bounds of the form $O(h)|u|_{2}$ in an energy norm. In addition, extensive numerical experiments show that they effectively reproduce boundary layers and internal layers caused by discontinuities on relatively coarse grids, without any requirements on alignment of flow and grid.
\end{abstract}

Key words. Convection-diffusion, streamline diffusion, crosswind diffusion, boundary layer, characteristic layer.

AMS(MOS) subject classifications. primary 65N30, 65F 10

1. Introduction. Consider the two-dimensional convection-diffusion equation

$$
\begin{aligned}
-\varepsilon \Delta u+\beta \cdot \nabla u & =f \text { in } \Omega, \\
u & =g \text { on } \partial \Omega,
\end{aligned}
$$

where $\beta=\left(\beta_{1}, \beta_{2}\right)$ is a flow velocity field, $\varepsilon$ is a diffusion or viscosity coefficient, and $f, g$ are given functions. For small values of $\varepsilon$, it is well known that standard Galerkin finite element discretizations yield inaccurate oscillatory solutions near boundary layers, and if $\varepsilon$ is decreased without proportional reduction of the discretization mesh size $h$, then these inaccuracies propagate into regions where the solution is smooth [9], [20, p. 259]. It is also known that these difficulties can be ameliorated using the streamline diffusion method [12], a Petrov-Galerkin finite element method in which the test functions are modified to produce a small amount of artificial diffusion in the direction of streamlines, thereby enhancing stability.

When solutions to (1)-(2) contain internal layers caused by discontinuities in boundary conditions, the approximate solution obtained by the streamline diffusion method may suffer from overshooting and undershooting along discontinuities [13], [14, p. 186]. "Shock-capturing" streamline diffusion methods add a discontinuity capturing term (in the test functions) to reduce the local oscillations [13, 25]. Unfortunately, the shock-capturing term depends on the unknown discrete solution and this leads to a nonlinear discrete system even though the original problem is linear. An alternative approach is to add artificial diffusion in the crosswind direction, although this may lead to overly diffuse numerical solutions in numerical experiments (see [16] and Section 5).

In this paper, we present some new variants of the streamline diffusion method that improve its performance. Our starting points are the papers of Roos [21], Stynes and

\footnotetext{
* Interdisciplinary Applied Mathematics Program, University of Maryland, College Park, MD 20742, email: yts@cs.umd.edu.

$\dagger$ Department of Computer Science and Institute for Advanced Computer Studies, University of Maryland, College Park, MD 20742, e-mail: elman@cs.umd.edu. This work was supported by U. S. National Science Foundation under grant DMS-9423133.
} 
Tobiska [24], and Fischer, et. al. [8]. The first two of these are concerned with necessary conditions on the structure of discretization operators for the convection-diffusion equation for convergence of the discrete solution to be uniform in $\varepsilon$. (Establishment of sufficient conditions appears to be a difficult problem.) In particular, Roos derives three conditions needed for uniform convergence in $L^{\infty}$ of order $h^{\gamma}, \gamma>0$, and Stynes and Tobiska show that two of Roos' conditions are needed for uniform convergence in $L^{2}$ of order $h^{\gamma}, \gamma>\frac{1}{2}$. The third paper [8] considers the problem of choosing the parameter that determines the amount of artificial diffusion included in the streamline diffusion discretization; it is shown that a good choice of this parameter leads to both small discretization errors and rapid convergence of certain iterative solvers for the discrete equations.

Here, we build on these points by introducing some two-parameter versions of streamline diffusion methods for bilinear (or linear) elements on uniform grids. The parameters determine the amount of artificial diffusion, but rather than being free parameters, they can be uniquely specified by requiring that the necessary conditions for $L^{2}$-uniform convergence established in [24] are satisfied. We present two versions of this idea that differ in their treatment of crosswind diffusion.

The contents of the rest of the paper are as follows. In Section 2, we outline some properties of the streamline diffusion method and variants that add crosswind diffusion. In Section 3, we summarize the necessary conditions for uniform convergence. In Section 4, we use the necessary conditions to derive the two new methods, and present an error analysis indicating that for linear or bilinear elements, they satisfy global error bounds in an energy norm of order $h|u|_{2}$. In Section 5 , we present the results of numerical experiments on benchmark problems that demonstrate the effectiveness of the new methods.

2. Streamline diffusion methods. For simplicity, we assume homogeneous Dirichlet boundary conditions on all boundaries; the ideas considered here generalize in a straightforward manner to other boundary conditions. Let $V_{h}^{0}=\{v \in$ $H^{1}(\Omega) \mid v=0$ on $\left.\partial \Omega\right\}$ denote the finite element space. Let $v_{\beta}=\beta \cdot \nabla v$ denote the derivative in the streamline direction. Let $\mathcal{T}=\tau_{h}$ denote a partitioning of $\Omega$. For our numerical examples, we will emphasize partitionings consisting of quadrilaterals; all the results of the paper apply naturally to triangles as well.

The streamline diffusion method (SD) is defined [14, p. 185] as: find $u^{h} \in V_{h}^{0}$ such that

$$
B_{s d}\left(u^{h}, v\right)=F_{v} \quad \forall v \in V_{h}^{0},
$$

where $B_{s d}(\cdot, \cdot)$ is the bilinear form

$$
\begin{aligned}
B_{s d}\left(u^{h}, v\right) & =B_{g}\left(u^{h}, v\right)+\delta_{s}\left(u_{\beta}^{h}, v_{\beta}\right) \\
F_{v} & =(f, v)+\delta_{s}\left(f, v_{\beta}\right),
\end{aligned}
$$

$B_{g}(u, v)=\varepsilon(\nabla u, \nabla v)+\left(u_{\beta}, v\right)$ denotes the bilinear form of the standard Galerkin method and $(\cdot, \cdot)$ denotes the usual scalar $L^{2}$ inner product. On a uniform grid with mesh parameter $h$ for which the mesh Péclet number $\frac{\|\beta\| h}{2 \varepsilon}$ is greater than 1 , the parameter $\delta_{s}$ is given by

$$
\delta_{s}=\omega_{s} h
$$


where $\omega_{s}$ is a fixed positive constant. ${ }^{1}$

The form $B_{s d}$ satisfies the coercivity condition

$$
B_{s d}(v, v) \geq \varepsilon\|\nabla v\|_{L^{2}(\Omega)}^{2}+\delta_{s}\left\|v_{\beta}\right\|_{L^{2}(\Omega)}^{2} \quad \forall v \in V_{h}
$$

in which the lower bound is positive in the limit $\varepsilon \searrow 0$. In particular, the finite element matrix has positive definite symmetric part and the discrete solution $u^{h}$ of (3) is unique. If $u$ is the strong solution and $f \in L^{2}(\Omega)$, then Axelsson [1] and Nävert [18] have shown that for a piecewise linear finite element space there is a constant $C$ (independent of $h, \delta_{s}$ and $\varepsilon$ ) such that

$$
\varepsilon^{1 / 2}\left\|\nabla\left(u-u^{h}\right)\right\|+\delta_{s}^{1 / 2}\left\|\left(u-u^{h}\right)_{\beta}\right\| \leq C\left(\varepsilon^{1 / 2} h+\delta_{s}^{1 / 2} h\right)|u|_{2},
$$

where $\|\cdot\|$ and $|\cdot|_{2}$ denote the usual $L^{2}$ norm and $H^{2}$ seminorm respectively. It is also shown in [2] that if $\delta_{s}=O(h), \varepsilon \leq c h$ and $-\nabla \cdot \beta \geq d_{0}>0$ for positive constant $d_{0}$, then the error for SD satisfies

$$
\left\|u-u^{h}\right\| \leq C h^{3 / 2}|u|_{2}
$$

this is shown without a duality argument or elliptic regularity.

In practice, when using SD to solve problems with characteristic internal and boundary layers, the choice of $\omega_{s}$ is crucial. Fischer et. al. [8] show that if $\varrho$ is the angle of flow to the horizontal, the choice

$$
\omega_{s}=\frac{1}{\|\beta\|}\left(\frac{1}{2}-\frac{\varepsilon}{h}|\cos \varrho|\right)
$$

is a good choice with respect to both clustering of the spectrum of the discrete operator and performance of an iterative solution algorithm.

Despite the success of SD, in practice, it suffers from excessive overshooting and undershooting of front following characteristics when discontinuities are present [13]. Johnson, Schatz and Wahlbin [15] introduced a modification of the SD discretization that improves its performance by adding artificial crosswind diffusion. The streamlinecrosswind diffusion method (SCD) as generalized by Lube [16] is given as follows: find $u \in V_{h}^{0}$ such that

$$
B_{s c d}\left(u^{h}, v\right)=F_{v} \quad \forall v \in V_{h}^{0},
$$

where

$$
B_{s c d}\left(u^{h}, v\right)=B_{s d}\left(u^{h}, v\right)+\left(\varepsilon_{m}-\varepsilon\right)\left(u_{\alpha}^{h}, v_{\alpha}\right),
$$

$\alpha=\left(-\beta_{2}, \beta_{1}\right)$ is the crosswind vector and the artificial crosswind diffusion is defined by

$$
\varepsilon_{m}= \begin{cases}\varepsilon & \text { for } \varepsilon \geq h^{3 / 2} \\ h^{3 / 2} & \text { for } \varepsilon<h^{3 / 2}\end{cases}
$$

\footnotetext{
${ }^{1}$ If $\mathcal{T}$ is not quasi-uniform or $\beta$ is a variable, then let $h$ be the diameter of local element and determine $\delta_{s}$ elementwise as in (6) (see [14, p. 186]).
} 
For this method with piecewise linear elements, pointwise error bounds of order $O\left(h^{2}|\log h|\right)$ have been obtained for special meshes in [27], where it is also shown that the width of the characteristic boundary layers and interior layers along streamlines are of order $h^{5 / 8} \log ^{2} h$. See also [22, pp. 229ff.] for discussion of such results.

Assume $\Omega=(0,1) \times(0,1)$ and a square mesh is generated by the nodes $\left(x_{i}, y_{j}\right)=$ ( $i h, j h)$, for $0 \leq i, j \leq N$, where $N$ is a positive integer and the mesh size $h=1 / N$. The SD discretization (3) leads to a system of linear equations with $(N-1)^{2}$ unknowns. Following the notation in [8], the resulting coefficient matrix can be expressed as

$$
\mathcal{A}_{s d}=\varepsilon \mathcal{H}+\mathcal{S}+\delta_{s} \mathcal{U}
$$

where

$$
\mathcal{H}_{i, j}=\left(\nabla \phi_{j}, \nabla \phi_{i}\right), \mathcal{S}_{i, j}=\left(\beta \cdot \nabla \phi_{j}, \phi_{i}\right), \mathcal{U}_{i, j}=\left(\beta \cdot \nabla \phi_{j}, \beta \cdot \nabla \phi_{i}\right)
$$

and $\left\{\phi_{i}\right\}_{i=1}^{(N-1)^{2}}$ are the finite element basis functions. The right hand side $b$ is given by

$$
b_{i}=\left(f, \phi_{i}+\delta_{s} \beta \cdot \nabla \phi_{i}\right) .
$$

If $\nabla \cdot \beta=0$, then for each basis function $\phi_{i}$ having value 0 on the boundary, it follows from integration by parts that

$$
\left(\beta \cdot \nabla \phi_{j}, \phi_{i}\right)=-\left(\beta \cdot \nabla \phi_{i}, \phi_{j}\right),
$$

that is, $\mathrm{S}$ is skew-symmetric. It is then easy to see that the symmetric part, $\varepsilon \mathcal{H}+\delta_{s} \mathcal{U}$, is positive definite. For constant $\beta$ and bilinear basis functions, the constituent 9 -point stencils are as follows:

$$
\begin{gathered}
\mathcal{H}: \\
\frac{1}{3}\left[\begin{array}{ccc}
-1 & -1 & -1 \\
-1 & 8 & -1 \\
-1 & -1 & -1
\end{array}\right] \\
\mathcal{S}: \\
\quad \frac{\beta_{1} h}{12}\left[\begin{array}{ccc}
-1 & 0 & 1 \\
-4 & 0 & 4 \\
-1 & 0 & 1
\end{array}\right]+\frac{\beta_{2} h}{12}\left[\begin{array}{ccc}
1 & 4 & 1 \\
0 & 0 & 0 \\
-1 & -4 & -1
\end{array}\right] \\
\mathcal{U}: \frac{1}{6}\left[\begin{array}{ccc}
-\beta_{1}^{2}-\beta_{2}^{2}+3 \beta_{1} \beta_{2} & 2 \beta_{1}^{2}-4 \beta_{2}^{2} & -\beta_{1}^{2}-\beta_{2}^{2}-3 \beta_{1} \beta_{2} \\
-4 \beta_{1}^{2}+2 \beta_{2}^{2} & 8\left(\beta_{1}^{2}+\beta_{2}^{2}\right) & -4 \beta_{1}^{2}+2 \beta_{2}^{2} \\
-\beta_{1}^{2}-\beta_{2}^{2}-3 \beta_{1} \beta_{2} & 2 \beta_{1}^{2}-4 \beta_{2}^{2} & -\beta_{1}^{2}-\beta_{2}^{2}+3 \beta_{1} \beta_{2}
\end{array}\right] .
\end{gathered}
$$

The coefficient matrix of the SCD discretization can be expressed as

$$
\mathcal{A}_{s c d}=\varepsilon \mathcal{H}+\mathcal{S}+\delta_{s} \mathcal{U}+\left(\varepsilon_{m}-\varepsilon\right) \mathcal{C}
$$

where

$$
\mathcal{C}_{i, j}=\left(\alpha \cdot \nabla \phi_{j}, \alpha \cdot \nabla \phi_{i}\right)
$$

The matrix $\varepsilon \mathcal{H}+\delta_{s} \mathcal{U}+\left(\varepsilon_{m}-\varepsilon\right) \mathcal{C}$ is also symmetric and positive definite, and the 9 -point stencil of $\mathcal{C}$ is given by

$$
\mathcal{C}: \frac{1}{6}\left[\begin{array}{ccc}
-\beta_{1}^{2}-\beta_{2}^{2}-3 \beta_{1} \beta_{2} & -4 \beta_{1}^{2}+2 \beta_{2}^{2} & -\beta_{1}^{2}-\beta_{2}^{2}+3 \beta_{1} \beta_{2} \\
2 \beta_{1}^{2}-4 \beta_{2}^{2} & 8\left(\beta_{1}^{2}+\beta_{2}^{2}\right) & 2 \beta_{1}^{2}-4 \beta_{2}^{2} \\
-\beta_{1}^{2}-\beta_{2}^{2}+3 \beta_{1} \beta_{2} & -4 \beta_{1}^{2}+2 \beta_{2}^{2} & -\beta_{1}^{2}-\beta_{2}^{2}-3 \beta_{1} \beta_{2}
\end{array}\right]
$$

In our numerical experiments, we find that this method dramatically reduces the oscillations of discrete solutions near boundary layers and internal layers, although there are still problems with smearing near sharp fronts (see Section 5). 
3. Necessary conditions. In this section, we outline some convergence criteria and analysis for the dependence of discrete solutions of (1)-(2) on the diffusion coefficient $\varepsilon$. In particular, let $\tau_{h}$ be a set of triangulations of $\Omega$ depending on a mesh parameter $h$. We will say that convergence of the discrete solution $u^{h}=u_{\varepsilon, h}$ is uniform in $\varepsilon$ if, for varying $\varepsilon$, and the parameter $h$ chosen such that $h / \varepsilon$ is constant, there is an error bound of the form

$$
\left\|u-u^{h}\right\| \leq C h^{\alpha},
$$

where $\alpha>0, C$ is independent of $\varepsilon$, and $\|\cdot\|$ is a norm. Convergence criteria of this type are considered in [10], [19], [21] and [24].

Here, we are concerned with necessary conditions for (17) to hold for the $L^{2}$ norm and $\alpha>\frac{1}{2}$. Assume $\beta_{1}$ and $\beta_{2}$ are constant and the discretization is on a uniform grid using bilinear or linear elements. The discrete problem then has the form

$$
\sum_{\nu, \mu=-1,0,1} a_{\nu, \mu} u_{i+\nu, j+\mu}^{h}=h \hat{f}_{i, j}, \quad \text { for } 1 \leq i, j \leq N,
$$

where $\hat{f}$ depends only on $f, u_{i, j}^{h}=u^{h}\left(x_{i}, y_{j}\right)$ and $\sum_{\nu, \mu=-1,0,1} a_{\nu, \mu}=0$.

THEOREM 3.1. [24] Let $f \in L^{2}(\Omega)$ such that the solution $u_{0}$ of the reduced problem

$$
\left\{\begin{array}{lll}
\beta \cdot \nabla u_{o}=f & \text { in } \Omega \\
u_{0}=0 & \text { on } \partial \Omega_{-}
\end{array}\right.
$$

is in $C^{1}(\bar{\Omega}) \cap C^{2}(\Omega)$ where $\partial \Omega_{-}=\{(x, y) \in \partial \Omega \mid \beta \cdot n<0, n$ is an outward unit normal vector $\}$. Assume that the scheme (18) yields a solution $u^{h}$ that satisfies

$$
\left\|u-u^{h}\right\| \leq C h^{\alpha} \quad \text { uniformly in } \varepsilon
$$

for $\alpha>\frac{1}{2}$. Then the coefficients of the scheme must satisfy

$$
\left(\sum_{\mu} a_{-1, \mu}\right) e^{\frac{-\beta_{1} h}{\varepsilon}}+\sum_{\mu} a_{0, \mu}+\left(\sum_{\mu} a_{1, \mu}\right) e^{\frac{\beta_{1} h}{\varepsilon}}=0
$$

and

$$
\left(\sum_{\nu} a_{\nu,-1}\right) e^{\frac{-\beta_{2} h}{\varepsilon}}+\sum_{\nu} a_{\nu, 0}+\left(\sum_{\nu} a_{\nu, 1}\right) e^{\frac{\beta_{2} h}{\varepsilon}}=0 .
$$

For the streamline diffusion method, the "stencil" coefficients $\left\{a_{\nu, \mu}\right\}$ depend linearly on the parameter $\omega_{s}$. In the case $\beta_{1}=\beta_{2}=\bar{\beta}$, the two conditions (20) and (21) are identical and they are satisfied by the choice

$$
\omega_{s}=\frac{1}{2 \bar{\beta}}\left(\operatorname{coth} \frac{\bar{\beta} h}{2 \varepsilon}-\frac{2 \varepsilon}{\bar{\beta} h}\right) .
$$

If the streamlines are aligned with the grid (e.g. $\beta_{2}=0$ ) then one of the equations $((21)$ in this case) is degenerate and the choice

$$
\omega_{s}=\frac{1}{2 \beta_{1}}\left(\operatorname{coth} \frac{\beta_{1} h}{2 \varepsilon}-\frac{2 \varepsilon}{\beta_{1} h}\right)
$$

satisfies (20). These results coincide with a well known "optimal value" in the onedimensional problem (see [6]) for which the discrete solution is exact at the nodes. Otherwise, if $\beta_{1} \neq \beta_{2}$, then (20) and (21) constitute two linear equations with a single unknown $\omega_{s}$, for which there is no solution [24]. Therefore, the streamline diffusion method does not satisfy these necessary conditions for uniform convergence. 
4. Two-parameter streamline diffusion schemes. Using the observations of the previous section as motivation, we now present two variants of the streamline diffusion method that contain two free parameters, which allow the necessary conditions of (20)-(21) to be satisfied. One of these techniques (the first) was presented for linear elements by Roos [21, p. 1465].2

In this section, we will assume that the velocity field $\beta$ satisfies $-\nabla \cdot \beta \geq d_{0} \geq 0$ for constant $d_{0}$. A case of primary interest is where both inequalities here are replaced by equality, as in the incompressible Navier-Stokes equations. We will also consider the case $d_{0}>0$; this is a technical assumption [2] which is used in the derivation of $L^{2}$-error bounds. (An alternative is to include a zero-order term in (1), see [4], [5], [26].) Experimental results described in Section 5 use benchmark problems in which $\beta$ is divergence free.

In our first variant, we add crosswind diffusion to the weak formulation, producing the new weak form (denoted SD-A)

$$
B_{s d a}\left(u^{h}, v\right)=F_{v} \quad \forall v \in V_{h}^{0},
$$

where

$$
B_{s d a}\left(u^{h}, v\right)=B_{s d}\left(u^{h}, v\right)+\delta_{c}\left(u_{\alpha}^{h}, v_{\alpha}\right)
$$

and $\delta_{c} \geq 0 .{ }^{3}$ For $-\nabla \cdot \beta \geq d_{0} \geq 0$, let

$$
\|v\|^{2}=\varepsilon\|\nabla v\|^{2}+\delta_{s}\left\|v_{\beta}\right\|^{2}+\delta_{c}\left\|v_{\alpha}\right\|^{2}+\frac{1}{2} d_{0}\|v\|^{2} .
$$

The stability of method (24) is a consequence of the following result.

Lemma 4.1. The bilinear form $B_{s d a}$ satisfies

$$
B_{s d a}(v, v) \geq\|v\|^{2} \quad \text { for any } v \in V_{h}^{0}(\Omega) .
$$

Proof. By Green's formula,

$$
0=\int_{\partial \Omega} v^{2} \beta \cdot n d S=\int_{\Omega} \nabla \cdot\left(v^{2} \beta\right) d \Omega=\int_{\Omega} \nabla \cdot \beta v^{2} d \Omega+2\left(v_{\beta}, v\right),
$$

and thus $\left(v_{\beta}, v\right)=-\frac{1}{2} \int_{\Omega} \nabla \cdot \beta v^{2} d \Omega \geq \frac{1}{2} d_{0}\|v\|^{2}$. Hence

$$
B_{s d a}(v, v) \geq \varepsilon\|\nabla v\|^{2}+\delta_{s}\left\|v_{\beta}\right\|^{2}+\delta_{c}\left\|v_{\alpha}\right\|^{2}+\frac{1}{2} d_{0}\|v\|^{2} .
$$

Therefore, the SD-A discretization has a unique solution.

We have the following error estimate, the proof of which follows [1], [15], [26].

THEOREM 4.2. Let $u$ be the solution of (1)-(2) with $g=0$ and $u \in H^{2}(\Omega) \cap$ $H_{0}^{1}(\Omega)$. Let $\beta \in W^{1, \infty}(\Omega)$ and either $\nabla \cdot \beta=0$ or $-\nabla \cdot \beta \geq d_{0}>0$, for constant $d_{0}$. If $u^{h}$ is the discrete solution obtained by $S D-A$ on either bilinear or linear elements, then the discretization error satisfies

$$
\left\|u-u^{h}\right\| \leq C\left(\varepsilon^{1 / 2} h+\delta_{s}^{1 / 2} h+\delta_{c}^{1 / 2} h+\delta_{s}^{-1 / 2} h^{2}+h^{2}+\delta_{c}\right)|u|_{2},
$$

for constant $C>0$.

\footnotetext{
${ }^{2}$ There it was observed that this scheme does not satisfy the conditions for $L^{\infty}$-uniform convergence when $\beta_{1} \neq \beta_{2}$.

${ }^{3}$ If $\mathcal{T}$ is not quasi-uniform or $\beta$ is a variable, then let $h$ be the diameter of local element and determine $\delta_{s}$ and $\delta_{c}$ elementwise as in SD.
} 
Proof. Let $\zeta=u^{I}-u$, where $u^{I}$ is the bilinear (or linear) interpolant of $u$. It follows that

$$
\begin{aligned}
&\|\zeta\| \leq c h^{2}|u|_{2}, \quad\|\nabla \zeta\| \leq c h|u|_{2} \\
&\|\zeta\| \leq c\left(\varepsilon^{1 / 2} h+\delta_{s}^{1 / 2} h+\delta_{c}^{1 / 2} h+h^{2}\right)|u|_{2}
\end{aligned}
$$

(see [14, p. 176], [22, p. 232]). Setting $\eta=u^{I}-u^{h}$ yields

$$
\|\eta\|^{2} \leq B_{s d a}(\eta, \eta)=B_{s d a}(\zeta, \eta)+B_{s d a}\left(u-u^{h}, \eta\right) .
$$

The quasi-orthogonality relation holds,

$$
B_{s d a}\left(u-u^{h}, v\right)=\operatorname{Per}(u, v) \text { for all } v \in V_{h}^{0},
$$

where $\operatorname{Per}(u, v)$ is the truncation error

$$
\operatorname{Per}(u, v)=\varepsilon\left(\Delta u, \delta_{s} v_{\beta}\right)+\delta_{c}\left(u_{\alpha}, v_{\alpha}\right)
$$

But Green's formula and the Poincaré inequality leads to

$$
\begin{aligned}
B_{s d a}\left(u-u^{h}, \eta\right) & \leq \varepsilon \delta_{s}^{1 / 2}\|\Delta u\| \delta_{s}^{1 / 2}\left\|\eta_{\beta}\right\|+\delta_{c}\left(\left\|\left(u_{\alpha}\right)_{\alpha}\right\|+\|\nabla \cdot \alpha\|_{\infty}\left\|u_{\alpha}\right\|\right)\|\eta\| \\
& \leq\left(\varepsilon \delta_{s}^{1 / 2}|u|_{2}+c \delta_{c}|u|_{2}\right)\|\eta\| \\
& \leq\left(\varepsilon \delta_{s}^{1 / 2}+c \delta_{c}\right)|u|_{2}\|\eta\|
\end{aligned}
$$

and

$$
B_{s d a}(\zeta, \eta)=\varepsilon(\nabla \zeta, \nabla \eta)+\delta_{s}\left(\zeta_{\beta}, \eta_{\beta}\right)+\delta_{c}\left(\zeta_{\alpha}, \eta_{\alpha}\right)-\left(\zeta, \eta_{\beta}\right)-\int_{\Omega}(\nabla \cdot \beta) \zeta \eta d \Omega .
$$

If $\nabla \cdot \beta=0$ (divergence free case), then

$$
\begin{aligned}
B_{s d a}(\zeta, \eta) & \leq \varepsilon\|\nabla \zeta\|\|\nabla \eta\|+\delta_{s}\left\|\zeta_{\beta}\right\|\left\|\eta_{\beta}\right\|+\delta_{c}\left\|\zeta_{\alpha}\right\|\left\|\eta_{\alpha}\right\|+\|\zeta\|\left\|\eta_{\beta}\right\| \\
& \leq\left(\varepsilon^{1 / 2}\|\nabla \zeta\|+\delta_{s}^{1 / 2}\left\|\zeta_{\beta}\right\|+\delta_{c}^{1 / 2}\left\|\zeta_{\alpha}\right\|+\delta_{s}^{-1 / 2}\|\zeta\|\right)\|\eta\| .
\end{aligned}
$$

If $-\nabla \cdot \beta \geq d_{0}>0$, then

$$
\int_{\Omega}(\nabla \cdot \beta) \zeta \eta d \Omega \leq c\|\zeta\|\|\eta\| \leq c^{\prime}\|\zeta\|\|\eta \eta\|
$$

and

$$
B_{s d a}(\zeta, \eta) \leq\left(\varepsilon^{1 / 2}\|\nabla \zeta\|+\delta_{s}^{1 / 2}\left\|\zeta_{\beta}\right\|+\delta_{c}^{1 / 2}\left\|\zeta_{\alpha}\right\|+\delta_{s}^{-1 / 2}\|\zeta\|+c^{\prime}\|\zeta\|\right)\|\eta\| .
$$

Use (28)-(29) to get

$$
B_{s d a}(\zeta, \eta) \leq c\left(\varepsilon^{1 / 2} h+\delta_{s}^{1 / 2} h+\delta_{c}^{1 / 2} h+\delta_{s}^{-1 / 2} h^{2}+h^{2}\right)|u|_{2}\|\eta\| .
$$

Combining (30), (31) and (32), gives

$$
\|\eta\| \leq c\left(\varepsilon^{1 / 2} h+\delta_{s}^{1 / 2} h+\delta_{c}^{1 / 2} h+\delta_{s}^{-1 / 2} h^{2}+h^{2}+\varepsilon \delta_{s}^{1 / 2}+\delta_{c}\right)|u|_{2} .
$$


Thus, using the triangle inequality, it follows that

$$
\begin{aligned}
\left\|u-u^{h}\right\| & \leq\|\eta\|+\|\zeta\| \\
& \leq C\left(\varepsilon^{1 / 2} h+\delta_{s}^{1 / 2} h+\delta_{c}^{1 / 2} h+\delta_{s}^{-1 / 2} h^{2}+h^{2}+\varepsilon \delta_{s}^{1 / 2}+\delta_{c}\right)|u|_{2} .
\end{aligned}
$$

As shown in [15], if $\varepsilon \leq c h$, then for the choices $\delta_{s}=O(h)$ and $\delta_{c}=O\left(h^{3 / 2}\right)$, all the terms of (27) depending on $\delta_{s}$ and $\delta_{c}$ are of same order of magnitude, and the error bound (27) implies that $\left\|u-u^{h}\right\| \leq C h^{3 / 2}|u|_{2}$. When $-\nabla \cdot \beta \geq d_{0}>0$, one can also get an $L^{2}$ bound $\left\|u-u^{h}\right\| \leq C h^{3 / 2}|u|_{2}$ which is the same estimate for SD shown in $[2]$.

We now consider a different approach for choosing $\delta_{s}$ and $\delta_{c}$ based on [24]. Suppose the discretization is on a uniform square mesh of width $h$ with bilinear shape functions. The resulting coefficient matrix has the form

$$
\mathcal{A}_{s d a}=\varepsilon \mathcal{H}+\mathcal{S}+\delta_{s} \mathcal{U}+\delta_{c} \mathcal{C},
$$

where $\mathcal{C}$ is as in (16). For $\beta_{1} \neq 0, \beta_{2} \neq 0$, the presence of two parameters in (33) allows us to impose conditions $(20)-(21)$. The result is a system of linear equations

$$
\begin{aligned}
\beta_{1}^{2} \delta_{s}+\beta_{2}^{2} \delta_{c} & =\frac{\beta_{1} h}{2} \operatorname{coth} \frac{\beta_{1} h}{2 \varepsilon}-\varepsilon, \\
\beta_{2}^{2} \delta_{s}+\beta_{1}^{2} \delta_{c} & =\frac{\beta_{2} h}{2} \operatorname{coth} \frac{\beta_{2} h}{2 \varepsilon}-\varepsilon .
\end{aligned}
$$

(The case $\beta_{1}=0$ or $\beta_{2}=0$ is addressed in Section 3.) For $\beta_{1} \neq \beta_{2}$, this system is nonsingular so the parameters $\delta_{s}, \delta_{c}$ are uniquely determined and have the values

$$
\begin{aligned}
\delta_{s} & =\frac{h}{|\beta|^{2}}\left(\frac{1}{2} \frac{\left.\beta_{1}^{3} \operatorname{coth} \frac{\beta_{1} h}{2 \varepsilon}-\beta_{2}^{3} \operatorname{coth} \frac{\beta_{2} h}{2 \varepsilon}-\varepsilon\right),}{\beta_{1}^{2}-\beta_{2}^{2}}-\right. \\
\delta_{c} & =\frac{h}{|\beta|^{2}}\left(\frac{1}{2} \frac{\beta_{1}^{2} \beta_{2} \operatorname{coth} \frac{\beta_{2} h}{2 \varepsilon}-\beta_{2}^{2} \beta_{1} \operatorname{coth} \frac{\beta_{1} h}{2 \varepsilon}}{\beta_{1}^{2}-\beta_{2}^{2}}-\varepsilon\right) .
\end{aligned}
$$

In case of $\beta_{1}=\beta_{2}$, we may choose the limit values of $\delta_{s}$ and $\delta_{c}$ as $\beta_{1} \rightarrow \beta_{2}$.

Let us compare these choices with $\delta_{s}$ defined by $(6),(8)$ and $\delta_{c}=h^{3 / 2}$. Assume $\varepsilon \ll h$ and $\beta_{1}=\cos \theta \neq 0, \beta_{2}=\sin \theta \neq 0$. The function $\operatorname{coth} \chi$ is very close to 1 for large $\chi$, and therefore the quantities in parentheses are essentially constant. Consequently, $\delta_{s}$ of $(36)$ and $\delta_{c}$ of $(37)$ are effectively proportional to $h$. This implies in particular that the asymptotic bound of $(27)$ is of size $O(h)$, larger than that of SCD. Figure 1 expands on this point. Plot (a) on the left compares the values of $\delta_{s} / h$ from (36) to $\omega_{s}$ from (8) and indicates that SD-A includes more diffusion in the upwind direction. Plot (b) compares $\delta_{c} / h$ to $h^{1 / 2}$ for several values of $h$; for moderate values of $h$ or values of $\theta$ near $0^{\circ}$ or $90^{\circ}$, the amount of added crosswind diffusion is comparable to that of SCD.

In the second two-parameter variant of SD, we introduce an extra parameter by modifying the contribution to the discrete diffusion operator in the coordinate dimension in which the flow is weaker. That is, the weak form (SD-B) is given by

$$
B_{s d b}\left(u^{h}, v\right)=F_{v} \quad \forall v \in V_{h}^{0},
$$




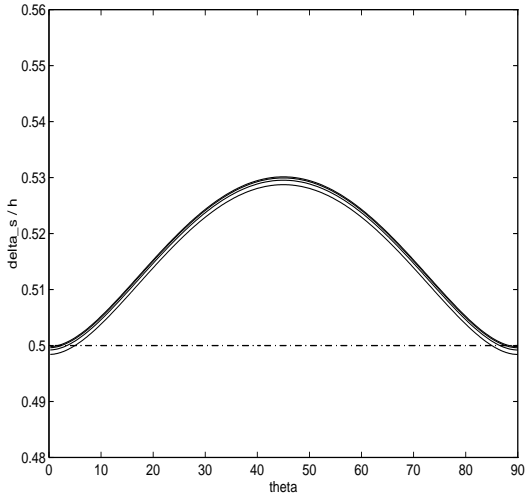

(a) Solid lines are $\delta_{s} / h$ from (36); dashdot line is $\omega_{s}$ from (8).

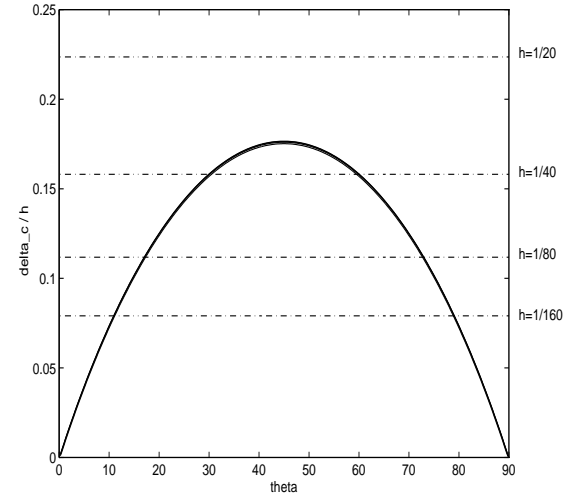

(b) Solid lines are $\delta_{c} / h$ from (37); dashdot lines are $h^{1 / 2}$.

FIG. 1. The values of $\delta_{s} / h, \delta_{c} / h$ for $\varepsilon=10^{-5}, \beta=(\cos \theta, \sin \theta), 0^{\circ} \leq \theta \leq 90^{\circ}$ and $h=$ $1 / 20,1 / 40,1 / 80,1 / 160$.

where

$$
\begin{gathered}
B_{s d b}\left(u^{h}, v\right)=B_{s d}\left(u^{h}, v\right)+\tilde{\varepsilon}\left(u_{\xi}^{h}, v_{\xi}\right), \\
\xi= \begin{cases}y & \text { if } 0<\left|\beta_{2}\right|<\left|\beta_{1}\right| \\
x & \text { if } 0<\left|\beta_{1}\right|<\left|\beta_{2}\right|,\end{cases}
\end{gathered}
$$

and $\tilde{\varepsilon} \geq 0$. If $\left|\beta_{1}\right|=\left|\beta_{2}\right|$ or the streamlines are aligned with the grid, then we use $\tilde{\varepsilon}=0$ and impose the necessary conditions using the SD method as in Section 3 .

The stability and error bounds for the SD-B scheme are summarized below. The proofs are analogous to those for SD-A and we omit them.

LEM A 4.3. If $-\nabla \cdot \beta \geq d_{0} \geq 0$ for constant $d_{0}$, then

$$
B_{s d b}(v, v) \geq \varepsilon\|\nabla v\|^{2}+\delta_{s}\left\|v_{\beta}\right\|^{2}+\tilde{\varepsilon}\left\|v_{\xi}\right\|^{2}+\frac{1}{2} d_{0}\|v\|^{2} \quad \text { for any } v \in H_{0}^{1}(\Omega)
$$

THEOREM 4.4. Let $u^{h}$ be the discrete solution obtained by SD-B on either bilinear or linear elements. Under the assumptions of Theorem 4.2, we have

$$
\left\|u-u^{h}\right\| \leq C\left(\varepsilon^{1 / 2} h+\delta_{s}^{1 / 2} h+\tilde{\varepsilon}^{1 / 2} h+\delta_{s}^{-1 / 2} h^{2}+h^{2}+\tilde{\varepsilon}\right)|u|_{2} .
$$

As above, the parameters $\delta_{s}$ and $\tilde{\varepsilon}$ can be used to satisfy the conditions $(20)-(21)$. The coefficient matrix derived from (39) is

$$
\mathcal{A}_{s d b}=\varepsilon \mathcal{H}+\mathcal{S}+\delta_{s} \mathcal{U}+\tilde{\varepsilon} \mathcal{P},
$$

where

$$
\mathcal{P}_{i, j}=\left(\frac{\partial \phi_{j}}{\partial \xi}, \frac{\partial \phi_{i}}{\partial \xi}\right)
$$


Consider the case when the streamlines are not aligned with the mesh, for example, $\beta_{1}>\beta_{2}>0$. Then the necessary conditions (20)-(21) lead to

$$
\begin{array}{r}
\left(-\varepsilon-\frac{\beta_{1} h}{2}-\delta_{s} \beta_{1}^{2}\right) e^{-\frac{\beta_{1} h}{\varepsilon}}+2\left(\varepsilon+\delta_{s} \beta_{1}^{2}\right)+\left(-\varepsilon+\frac{\beta_{1} h}{2}-\delta_{s} \beta_{1}^{2}\right) e^{\frac{\beta_{1} h}{\varepsilon}}=0 \\
\left(-\tilde{\varepsilon}-\varepsilon-\frac{\beta_{2} h}{2}-\delta_{s} \beta_{2}^{2}\right) e^{-\frac{\beta_{2} h}{\varepsilon}}+2\left(\tilde{\varepsilon}+\varepsilon+\delta_{s} \beta_{2}^{2}\right)+\left(-\tilde{\varepsilon}-\varepsilon+\frac{\beta_{2} h}{2}-\delta_{s} \beta_{2}^{2}\right) e^{\frac{\beta_{2} h}{\varepsilon}}=0 .
\end{array}
$$

That is,

$$
\begin{aligned}
\delta_{s} & =\frac{h}{2 \beta_{1}} \operatorname{coth} \frac{\beta_{1} h}{2 \varepsilon}-\varepsilon, \\
\tilde{\varepsilon} & =\frac{\beta_{2} h}{2} \operatorname{coth} \frac{\beta_{2} h}{2 \varepsilon}-\delta_{s} \beta_{2}^{2}-\varepsilon .
\end{aligned}
$$

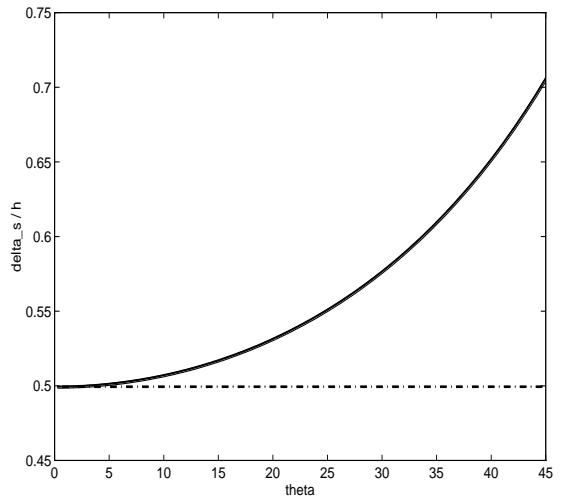

(a) Solid lines are $\delta_{s} / h$ from (41); dashdot line is $\omega_{s}$ from (8).

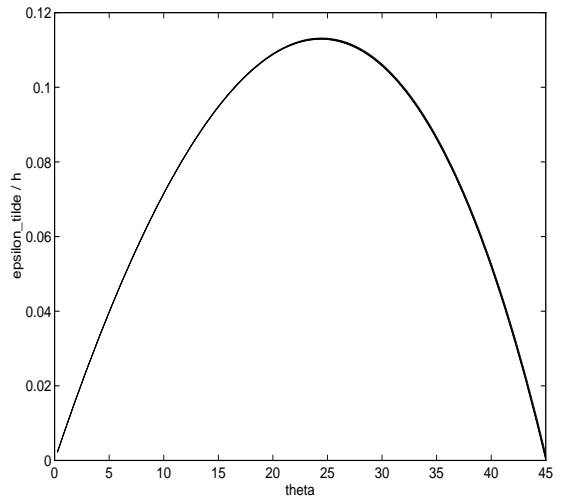

(b) $\tilde{\varepsilon} / h$ obtained by (42)

FIG. 2. The values of $\delta_{c}$ and $\tilde{\varepsilon}$ for $\varepsilon=10^{-5}, \beta=(\cos \theta, \sin \theta), 0^{\circ} \leq \theta \leq 45^{\circ}$ and $h=$ $1 / 20,1 / 40,1 / 80,1 / 160$.

Let us compare these choices with $\delta_{s}$ defined by $(6)$ and (8). In Figure 2 , for $\varepsilon=$ $10^{-5}, \beta=(\cos \theta, \sin \theta), 0^{\circ} \leq \theta \leq 45^{\circ}$ and various mesh sizes $h=1 / 20,1 / 40,1 / 80,1 / 160$, Plot (a) on the left compares the value of $\delta_{s} / h$ from (41) to $\omega_{s}$ from (8) and indicates that SD-B also includes more diffusion in the upwind direction. Plot (b) shows the values of $\tilde{\varepsilon} / h$ obtained by (42) which determines the amount of crosswind-like diffusion. We see that $\tilde{\varepsilon}$ dissipates when the streamlines are aligned with the mesh or $\beta_{1}=\beta_{2}$.

Remark 4.1 We are using the necessary conditions for the uniform convergence as a means of specifying the parameters in these discretizations. The conditions are not known to be sufficient for uniform convergence and we are not considering this issue here. Cf. [22, pp. 273ff.] for other discretizations that display uniform convergence.

5. Numerical experiments. The analytic results used and cited in Section 4 are for smooth problems, i.e., $u \in H^{2}(\Omega)$. However, the real need for streamline diffusion methods occurs for problems with steep boundary layers or discontinuities. In this section, we present the results of numerical experiments that show the performance of the new streamline diffusion methods for such problems and compare it to 
that of the classical methods SD and SCD. We use three test problems. One has a known solution given by the sum of two one-dimensional examples and containing downstream boundary layers, one has a downstream boundary layer and a characteristic internal layer, and one has variable flow. All experiments use bilinear shape functions on square elements on a uniform $N \times N$ element grid with $h=1 / N$, and they were performed with MATLAB Version $4.2 \mathrm{c}$ on a SUN SPARC-20 workstation. The coefficient $\delta_{s}=\omega_{s} h$ of the streamline diffusion term for both SD and SCD was chosen using (8).
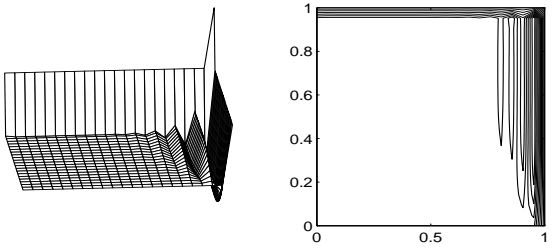

(a) SD
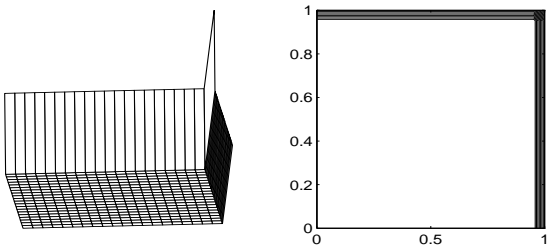

(c) SD-A
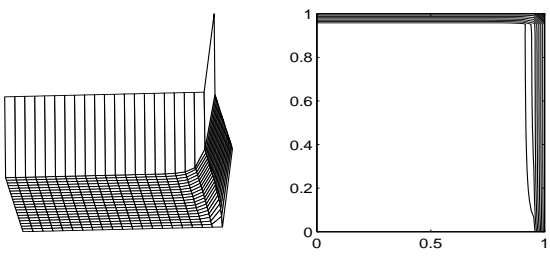

(b) SCD
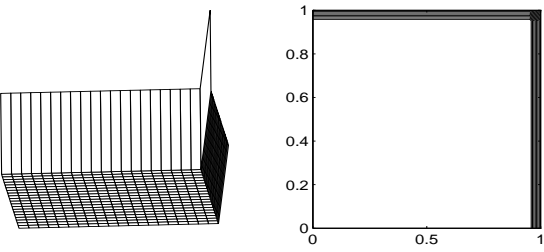

(d) SD-B

FIG. 3. Numerical solutions for problem 1 for $\varepsilon=10^{-4}, \theta=75^{\circ}, h=1 / 20$.

TABLE 1

Maximum absolute errors at the nodes for $h=1 / 20$.

\begin{tabular}{|l|c|c|c|c|c|}
\hline$\theta$ & $\varepsilon$ & SD & SCD & SD-A & SD-B \\
\hline \multirow{2}{*}{0} & $1 . e-2$ & $8.58 e-3$ & $8.55 e-3$ & $1.3 e-15$ & $1.1 e-15$ \\
& $1 . e-4$ & $4.3 e-15$ & $2.8 e-15$ & $4.3 e-15$ & $4.3 e-15$ \\
\hline \multirow{2}{*}{$15^{\circ}$} & $1 . e-2$ & $1.81 e-2$ & $2.55 e-2$ & $3.9 e-16$ & $2.2 e-16$ \\
& $1 . e-4$ & $5.97 e-1$ & $3.11 e-1$ & $9.5 e-17$ & $2.7 e-16$ \\
\hline \multirow{2}{*}{$45^{\circ}$} & $1 . e-2$ & $1.94 e-2$ & $2.68 e-2$ & $6.9 e-17$ & $9.7 e-17$ \\
& $1 . e-4$ & $3.89 e-1$ & $2.11 e-2$ & $3.3 e-16$ & $1 . e-146$ \\
\hline
\end{tabular}

Problem 1: Downstream boundary layers/analytic solution. We first consider the problem with exact known solution given by

$$
u(x, y)=\frac{e^{\beta_{1} x / \varepsilon}-1}{e^{\beta_{1} / \varepsilon}-1}+\frac{e^{\beta_{2} y / \varepsilon}-1}{e^{\beta_{2} / \varepsilon}-1},
$$

where $\left(\beta_{1}, \beta_{2}\right)=(\cos \theta, \sin \theta)$ for $0^{\circ}<\theta<90^{\circ}$. (For $\theta=0^{\circ}, u(x, y)=\frac{e^{x / \varepsilon}-1}{e^{1 / \varepsilon}-1}+y$, the limit value.) The Dirichlet boundary conditions and right hand side $f$ are determined 
from the exact solution. Figure 3 depicts the numerical solutions for $\varepsilon=10^{-4}, \theta=75^{\circ}$ and $h=1 / 20$. The results indicate that the two new schemes reduce the oscillations or excess diffusivity exhibited by SD and SCD, respectively. Indeed, for these problems we find that the new schemes reproduce the exact solution at the nodes essentially to within machine precision, as shown in Table 1.

Problem 2: Characteristic and downstream boundary layers. This problem was first considered in [12] for studying a downstream boundary layer and a characteristic internal layer that propagates along the characteristics when inflow boundary conditions are discontinuous. Assume the velocity field $\beta$ is given by $(\cos \theta, \sin \theta)$, and boundary values are as follows:

$$
u= \begin{cases}1 & \text { if } 0 \leq y<1 / 2, x=0 \text { or } y=0,0 \leq x<1 \\ 0 & \text { otherwise. }\end{cases}
$$

The reduced problem (19) has discontinuous solution

$$
u=\left\{\begin{array}{ll}
1 & y<\frac{\beta_{2}}{\beta_{1}} x+\frac{1}{2} \\
0 & y>\frac{\beta_{2}}{\beta_{1}} x+\frac{1}{2}
\end{array} .\right.
$$

For $\varepsilon>0$, there is an internal layer of width $O(\sqrt{\varepsilon})$ across the characteristic $y=$ $\frac{\beta_{2}}{\beta_{1}} x+\frac{1}{2}$, and a boundary layer of width $O(\varepsilon)$ at $x=1[7]$.
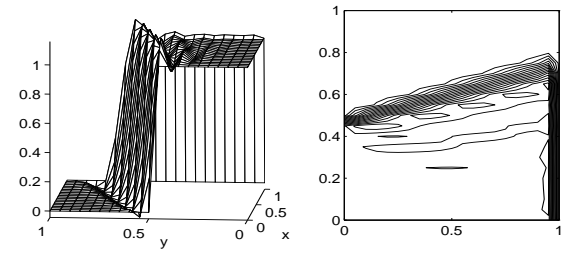

(a) SD
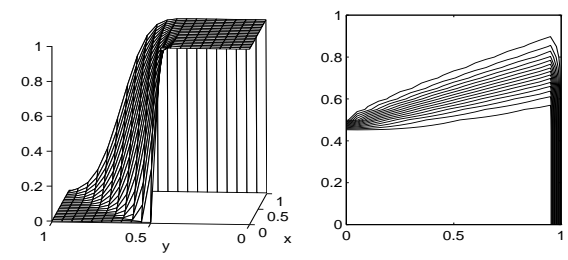

(c) SD-A
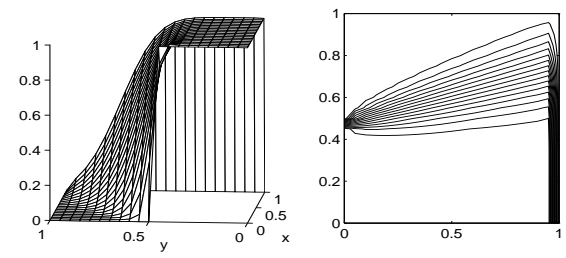

(b) SCD
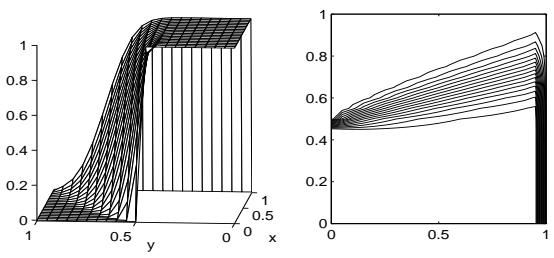

(d) SD-B

FIG. 4. Numerical solutions and contours for $\varepsilon=10^{-5}, h=1 / 20$ and $\theta=15^{\circ}$.

We first examine the accuracy with which the internal layer is approximated by the various discretizations. Figure 4 depicts the three-dimensional structure and contour plots of the numerical solutions obtained by the four methods tested, for $\varepsilon=10^{-4}, h=$ $1 / 20$ and $\beta=\left(\cos 15^{\circ}, \sin 15^{\circ}\right)$. Figure 5 shows contour plots for $\varepsilon=10^{-5}$ and several mesh sizes. It is evident that the solutions obtained by SD oscillate around the internal layer and those obtained by SCD are overly diffuse. The two new variants appear to 

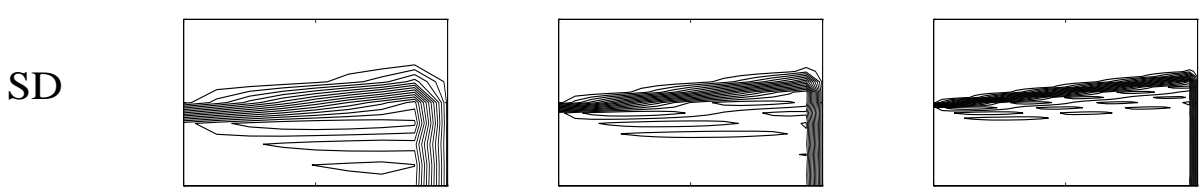

SCD
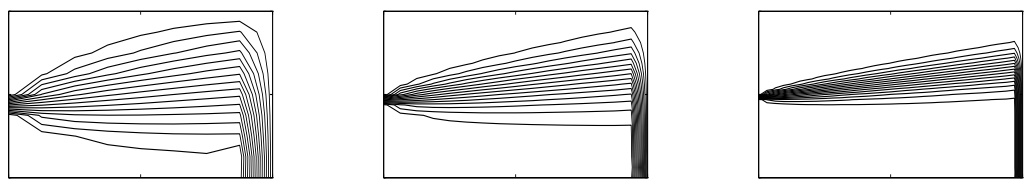

SD-A
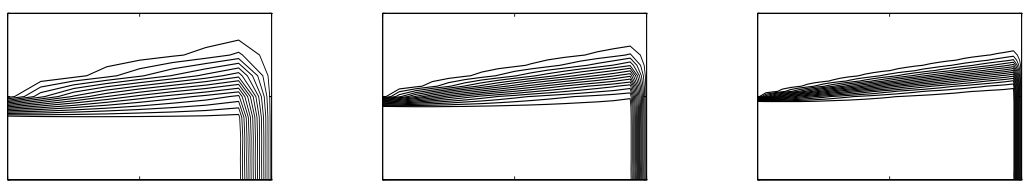

SD-B
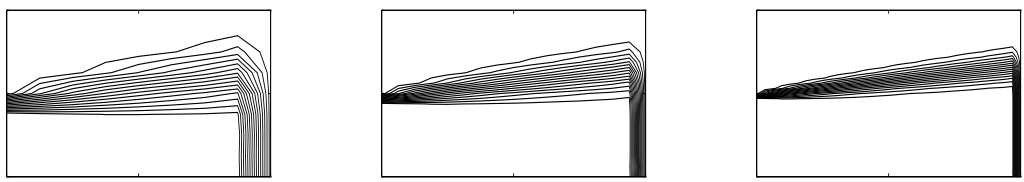

(a) $8 \times 8$ grids

(b) $16 \times 16$ grids

(c) $32 \times 32$ grids

FIG. 5. Contour plots showing numerical internal and boundary layers for $\varepsilon=10^{-5}$ and $\theta=15^{\circ}$.

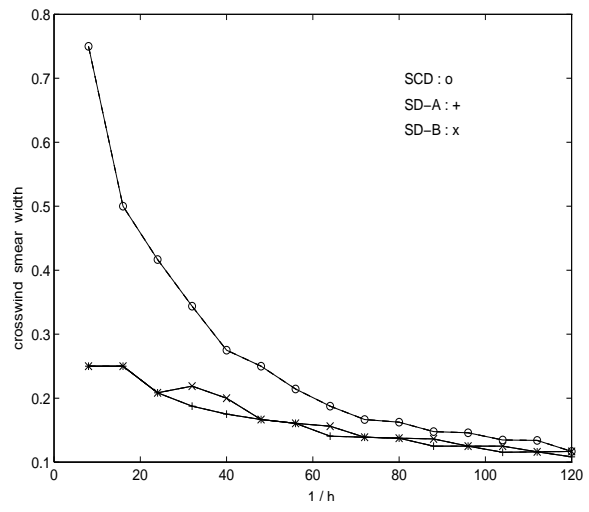

FIG. 6. The crosswind smear width $(\Delta y)$ when mesh is refined and $\varepsilon=10^{-5}, \sigma=10^{-3}$ and $\theta=15^{\circ}$

TABLE 2

The measure of overshoots and undershoots for $\varepsilon=10^{-5}$.

\begin{tabular}{|l|c|r|c|r|r|}
\hline$h$ & shooting & \multicolumn{1}{|c|}{ SD } & SCD & \multicolumn{1}{c|}{ SD-A } & \multicolumn{1}{c|}{ SD-B } \\
\hline \multirow{2}{*}{$1 / 16$} & $u_{+}$ & $1.12 e-1$ & 0 & $5.49 e-5$ & 0 \\
& $u_{-}$ & $-3.44 e-2$ & 0 & $-1.20 e-4$ & $-2.79 e-4$ \\
\hline \multirow{2}{*}{$1 / 32$} & $u_{+}$ & $1.10 e-1$ & 0 & $9.54 e-7$ & $1.3 e-15$ \\
& $u_{-}$ & $-4.16 e-2$ & 0 & $-6.02 e-6$ & $-2.29 e-5$ \\
\hline \multirow{2}{*}{$1 / 64$} & $u_{+}$ & $9.78 e-2$ & $3.6 e-12$ & $3.07 e-9$ & $2.7 e-15$ \\
& $u_{-}$ & $-3.44 e-2$ & $-6.8 e-14$ & $-5.45 e-8$ & $-3.52 e-7$ \\
\hline
\end{tabular}


produce a more accurate representation of the internal layer. We can quantify this statement as follows. Let

$y_{u}=\min _{0 \leq y \leq 1}\left\{y \mid u^{h}(0.5, y) \geq \sigma\right\}, y_{l}=\max _{0 \leq y \leq 1}\left\{y \mid u^{h}(0.5, y) \leq 1-\sigma\right\}, \quad$ for small $\sigma>0$.

Then $\Delta y=y_{u}-y_{l}$ is a measure of the width of the numerical internal layer and the effect of crosswind smearing at $x=0.5$. Figure 6 plots this quantity for various mesh sizes and $\sigma=10^{-3}$. The results indicate that the two-parameter schemes introduce less crosswind smearing than SCD. Moreover, the lower-order $O(h)$ asymptotic bound (Theorem 4.2) obtained for smooth problems does not appear to have a deleterious effect as $h \searrow 0$ in this nonsmooth example.

It is known that SD produces overshooting and undershooting about sharp layers [13]. We can use a similar device to examine the size of these overshoots and undershoots. Let

$$
u_{+}=\max _{0 \leq y \leq 1}\left\{u^{h}(0.5, y)-1\right\}, \quad u_{-}=\min _{0 \leq y \leq 1}\left\{u^{h}(0.5, y)\right\} .
$$

Thus $u_{+}, u_{-}$give a measure of the sizes of overshooting and undershooting respectively, at $x=0.5$. Table 2 shows the effectiveness of SD-A and SD-B in this measure.

Remark 5.1 These results for $\beta=\left(\cos 15^{\circ}, \sin 15^{\circ}\right)$ are representative of our experience with other velocity fields $\beta=(\cos \theta, \sin \theta)$ for $\theta \in\left(-90^{\circ}, 90^{\circ}\right)$.

Problem 3: Variable flow field. For variable flows, we can define local (to element) values of parameters determining the amounts of artificial diffusion. We follow the approach given in [3]: on any element $\tau$, let $\left(x_{\tau}, y_{\tau}\right)$ denote the element center, let $\beta_{\tau}=\beta\left(x_{\tau}, y_{\tau}\right)$, and let these constant values be used to define the parameters in formulas (8), (36)-(37) and (41)-(42) in the local matrix computations associated with the element $\tau$. Note that a similar approach could also be used for irregular grids.

For our third benchmark problem, with variable flow, we consider two variants of the "IAHR/CEGB" workshop problem [23] in common use for testing discretization strategies (see e.g. [11], [17]). As in [23], let the domain be the rectangular region

$$
\Omega=\{(x, y) \mid-1<x<1,0<y<1\},
$$

and the velocity field be

$$
\beta=\left(2 y\left(1-x^{2}\right),-2 x\left(1-y^{2}\right)\right) .
$$

The inflow boundary is the interval $\{(x, 0) \mid-1 \leq x<0\}$, and Dirichlet conditions specified there represent an inlet temperature which is convected in a circular flow to the outflow boundary $\{(x, 0) \mid 0<x \leq 1\}$, where natural boundary conditions

$$
\frac{\partial u(x, 0)}{\partial n}=0, \quad \text { for } 0<x \leq 1
$$

are assigned. For both our variants, Dirichlet boundary conditions are given on the remainder of $\partial \Omega$.

The first choice is defined by values

$$
u(x, 0)=1+\tanh (10+20 x)
$$



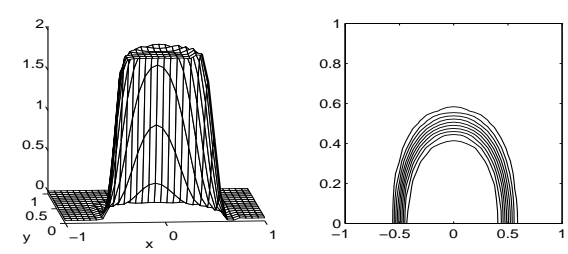

(a) SD
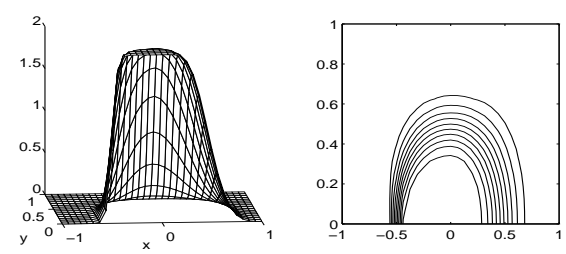

(c) SD-A
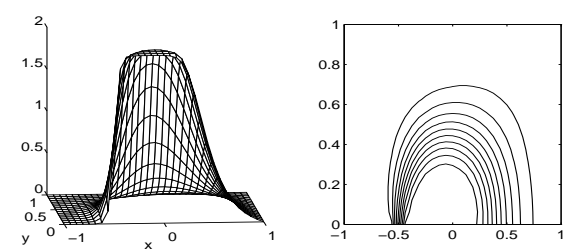

(b) SCD
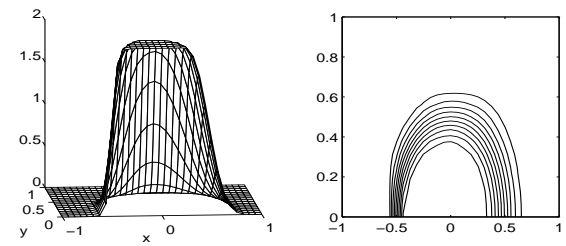

(d) SD-B

FIG. 7. Numerical solutions and contours for Problem 3, first variant with $\varepsilon=10^{-5}$ and $h=1 / 16$.
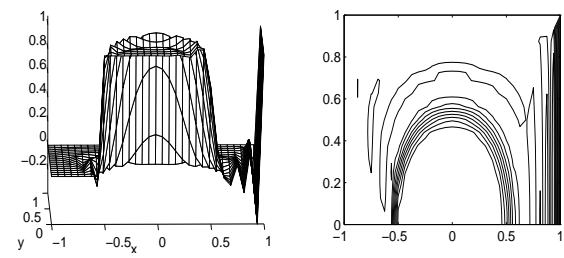

(a) SD
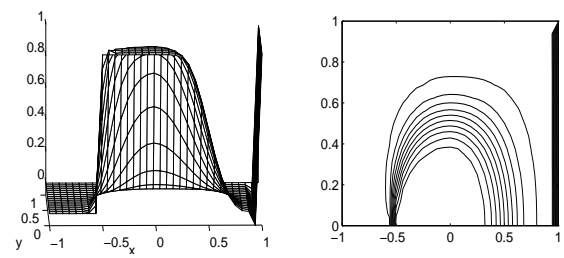

(c) SD-A
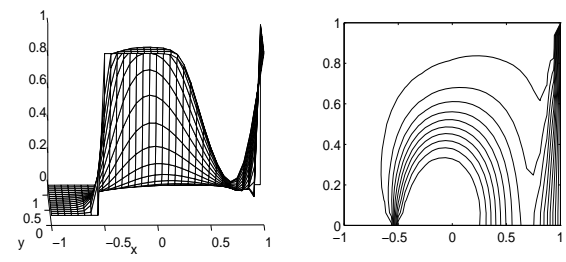

(b) SCD
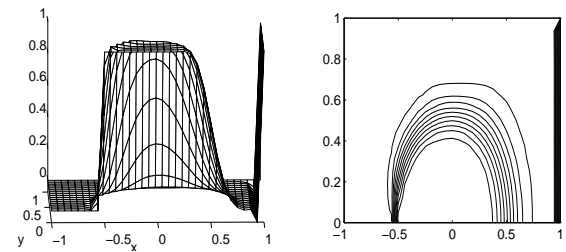

(d) SD-B

FIG. 8. Numerical solutions and contours for Problem 3, second variant with $\varepsilon=10^{-5}$ and $h=1 / 16$. 
at the inlet together with $u=0$ at $x= \pm 1$ and $y=1$. This is essentially the problem of [23] and it has also been examined in [11]. It contains a fairly smooth characteristic internal layer and no boundary layer. Representative pictures of the three-dimensional structure and contour plots of the numerical solutions obtained by the four methods tested, for $\varepsilon=10^{-5}$ and $h=1 / 16$, are shown in Figure 7 . (The three-dimensional plots are rotated $180^{\circ}$ to give a clearer picture of the layer.) Our second variant of this problem uses the value $u=1$ (a hot wall) at $x=1$ as in [17]; this introduces a thin boundary layer at the right boundary. In addition, we add a discontinuity in the inlet profile

$$
u(x, 0)= \begin{cases}0 & -1 \leq x<-0.5 \\ 1 & -0.5 \leq x \leq 0 .\end{cases}
$$

The homogeneous Dirichlet conditions at $x=-1$ and $y=1$ remain intact. The results for this example, again for $\varepsilon=10^{-5}, h=1 / 16$, are shown in Figure 8 .

Consideration of Figure 7 shows that for the (relatively smooth) example determined by (43) and the homogeneous condition at $x=1$, the best solution is obtained by pure streamline-diffusion (SD). The new variants SD-A and SD-B produce solutions that are somewhat overdiffuse, but quite a bit less so than SCD. We also remark that the solution obtained by the Galerkin method without any upwinding is qualitatively similar to that obtained by SD. Figure 8 shows that the situation is different when discontinuities are present. None of the methods eliminate oscillations completely (showing that none are monotonic), but the new methods clearly improve the accuracy of the solution near the boundary layer. They also produce more accurate approximations to the internal layer than SCD, and, in contrast with those obtained by SD, these components of the solutions are nonoscillatory. There may still be some smearing of the crosswind layer; here SD-B seems to be slightly more effective than SD-A.

\section{REFERENCES}

[1] O. Axelsson. On the numerical solution of convection dominated convection-diffusion problems. In K. I. Gross, editor, Mathematics Methods in Energy Research, pages 3-21. SIAM, Philadelphia, 1984.

[2] O. Axelsson, V. Eijkhout, B. Polman, and P. Vassilevski. Incomplete block-matrix factorization iterative methods for convection-diffusion problems. BIT, 29:867-889, 1989.

[3] A. Brooks and T. Hughes. Streamline upwind/Petrov-Galerkin formulations for convection dominated flows with particular emphasis on the incompressible Navier-Stokes equations. Comp. Meth. Appl. Mech. Engng., 32:199-259, 1982.

[4] C. Johnson and U. Nävert. An analysis of some finite element methods for advection-diffusion problems. In O. Alexsson, L.S. Frank, and A. van der Sluis, editors, Analytical and Numerical Approaches to Asymptotic Problem in Analysis. North-Holland, Amsterdam, 1981.

[5] C. Johnson and U. Nävert and J. Pitkäranta. Finite element methods for linear hyperbolic problems. Comp. Meths. Appl. Mech. Engrg., 45:285-312, 1984.

[6] I. Christie, D.F. Griffiths, A.R. Mitchell, and O.C. Zienkiewicz. Finite element methods for second order differential equations with significant first derivatives. Int. J. Numer. Meth. Engrg., 10:1389-1396, 1976.

[7] W. Eckhaus. Boundary layers in linear elliptic singular perturbation problems. SIAM Review, 14:225-270, 1972.

[8] B. Fischer, A. Ramage, D. Silvester, and A.J. Wathen. Towards parameter-free streamline upwinding for advection-diffusion problems. Technical Report 37, Department of Mathematics, University of Strathclyde, 1996. 
[9] P. M. Gresho and R. L. Lee. Don't suppress the wiggles - they're telling you something. Computers and Fluids, 9:223-253, 1981.

[10] A. F. Hegarty, E. O'Riordan, and M. Stynes. A comparison of uniformly convergent difference schemes for two-dimensional convection-diffusion problems. J. Comp. Phys., 105:24-32, 1993.

[11] P. W. Hemker. Mixed defect correction iteration for the accurate solution of the convection diffusion equation. In W. Hackbusch and U. Trottenberg, editors, Multi-grid Methods, pages 485-501. Springer-Verlag, Berlin, 1982.

[12] T. J. Hughes and A. Brooks. A multidimensional upwind scheme with no crosswind diffusion. In T. J. Hughes, editor, Finite Element Methods for Convection Dominated Flows. AMSE, New York, 1979.

[13] T. J. R. Hughes, M. Mallet, and A. Mizukami. A new finite element formulation for computational fluid dynamics: II. Beyond SUPG. Comp. Meths. Appl. Mech. Engrg., 54:341-355, 1986.

[14] C. Johnson. Numerical Solution of Partial Differential Equations by the Finite Element Method. Cambridge University Press, New York, 1987.

[15] C. Johnson, A.H. Schatz, and L.B. Wahlbin. Crosswind smear and pointwise errors in streamline diffusion finite element methods. Math. Comp., 49:25-38, 1987.

[16] G. Lube. An asymptotically fitted finite element method for convection dominated convectiondiffusion-reaction problems. Math. Mech., 72:189-200, 1992.

[17] K. W. Morton, editor. Numerical Solution of Convection-Diffusion Problems. Chapman \& Hall, London, 1996.

[18] U. Nävert. A Finite Element Method for Convection-Diffusion Problems. PhD thesis, Chalmers University of Technology, 1982.

[19] E. O’Riordan and M. Stynes. A globally uniformly convergent finite element method for a singularly perturbed elliptic problem in two dimensions. Math. Comp., 57:47-62, 1991.

[20] A. Quarteroni and A. Valli. Numerical Approximation of Partial Differential Equations. Springer-Verlag, New York, 1994.

[21] H. G. Roos. Necessary convergence conditions for upwind schemes in the two-dimensional case. Int. J. Numer. Meth. Engrg., 21:1459-1469, 1985.

[22] H.G. Roos, M. Stynes, and L. Tobiska. Numerical Methods for Singularly Perturbed Differential Equations. Springer-Verlag, New York, 1996.

[23] R. M. Smith and A. G. Hutton. The numerical treatment of advection - a performance comparison of current methods. Numer. Heat Transfer, 5:439-461, 1982.

[24] M. Stynes and L. Tobiska. Necessary $L^{2}$-uniform convergence conditions for difference schemes for two dimensional convection- diffusion problems. Computers Math. Applic., 29:45-53, 1995.

[25] A. Szepessy. Convergence of a Finite Element Method for Hyperbolic Conservation Laws. PhD thesis, Chalmers University of Technology, 1989.

[26] G. Zhou. How accurate is the streamline diffusion finite element method? Math. Comp., 66:3144, 1997.

[27] G. Zhou and R. Rannacher. Pointwise superconvergence of the streamline diffusion finite element method. Numer. Methods Partial Diff. Equations, 12:123-145, 1996. 